\title{
Long-term behavior of non-ferrous metal price models with jumps
}

\author{
Jun Peng ${ }^{1,2,3^{*}}$ and Jianbai Huang ${ }^{1,3}$
}

"Correspondence:

pengjun0825@163.com

${ }^{1}$ Business School, Central South

University, Changsha, Hunan

410083, China

${ }^{2}$ School of Mathematics and

Statistics, Central South University,

Changsha, Hunan 410083, China

Full list of author information is

available at the end of the article

\begin{abstract}
In this paper, we study the long-term behavior of a class of stochastic non-ferrous metal prices with jumps. Suppose that $X(t)$ is a stochastic model for some metal price with Poisson jumps. For a suitable $\mu \geq 1$, we prove that $t^{-\mu} \int_{0}^{t} X(s) d s$ converges almost surely as $t \rightarrow \infty$. Finally, the model is applied to forecast the behavior of a two-factor affine model.
\end{abstract}

MSC: $60 \mathrm{H} 15 ; 86 \mathrm{~A} 05 ; 34 \mathrm{D} 35$

Keywords: long-term behavior; jump; geometric Brownian motion; convergence

\section{Introduction}

Non-ferrous metal resources commodity producers, consumers and investors face problems resulting from the great variability in metal prices over time. The metal price fluctuations affect metal consumers by increasing or decreasing production cost. Obviously, the consumer wants the price to be as low as possible. Therefore, the metal price should not be too high to lose the clients because of the drastic competition arising from the open market. On the other hand, if the price is lower than the estimated random price in order to cover expenses and to hold some reserves, the companies would go bankrupt. In this light, it is very useful to study and to model the long-time behavior in a mathematical way. As discussed by Ahrens and Sharma [1] (elsewhere [2-5]), natural resources commodity prices exhibit stochastic trends. In order to capture the properties of empirical data, Brennan and Schwartz [6] proposed a geometric Brownian motion (GBM) model for forecasting natural resources commodity prices $Y_{t}$ :

$$
d Y_{t}=\theta Y_{t} d t+\sigma Y_{t} d W_{t}, \quad t \geq 0, \quad y_{0}=0,
$$

where $d W_{t}$ is the increment in a Gauss-Wiener process with drift $\theta$ and instantaneous standard deviation $\sigma$. A geometric Brownian motion or exponential Brownian motion is a continuous-time stochastic process in which the logarithm of the randomly varying quantity follows a Brownian motion or a Wiener process. It is applicable to mathematical modeling of some phenomena in financial markets. GBM is used as a mathematical model in financial markets and in forecasting prices. The GBM formula means that in any interval of time, prices will never be negative and can either go up or down randomly as a function of their volatility'.

c) 2014 Peng and Huang; licensee Springer. This is an Open Access article distributed under the terms of the Creative Commons Attribution License (http://creativecommons.org/licenses/by/2.0), which permits unrestricted use, distribution, and reproduction in any medium, provided the original work is properly cited. 


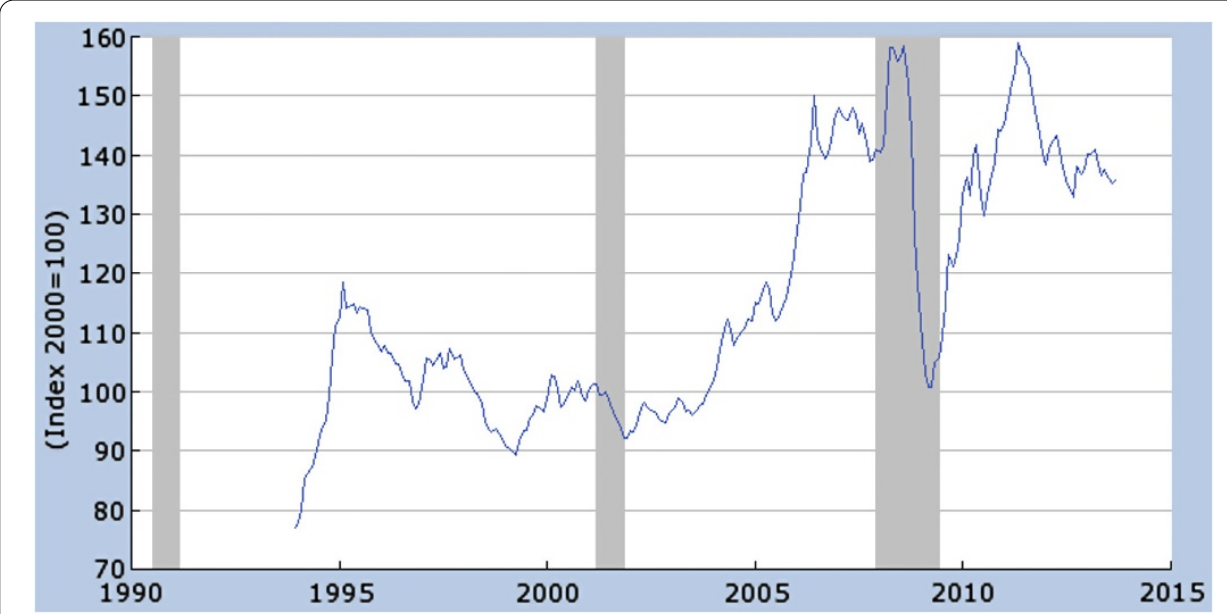

Figure 1 Monthly data for the price of aluminum and articles thereof (IP76) from January 1994 to October 2013. Shaded areas indicate US recessions. We quoted the data from US Department of Labor: Bureau of Labor Statistics.

One generalization of the GBM model is to use regime switching such as in $[7,8]$, to name a few. Hamilton [9] characterizes business cycles as periods of discrete regime shifts, i.e., recessions are characterized belonging to one regime and expansions to another in a Markov-switching process. The main advantage of the Markov-switching space state model over the standard GARCH model is that in the case of the latter the unconditional variance is constant, while in the former the variance changes according to the state of the economy. However, the Markov-switching model has been criticized because it lacks transparency, is less robust and is difficult to apply [10].

On the other hand, various economic shocks, news announcement, government policy changes, market demands may affect the metal price in a sudden way and generate nonferrous metal price jumps. As indicated in Figure 1, the jumps do exist in the aluminum prices realization. This paper introduces a new comprehensive version of the long-term trend reverting jump and diffusion model. The behavior of historical non-ferrous metal prices includes three different components: long-term reversion, diffusion and jump. The long-term behavior of stochastic interest rate models was discussed in [11-13], where they studied the Cox-Ingersoll-Ross model.

In this paper, in order to incorporate sudden jumps in spot market, we consider the stochastic metal price model with jumps in the form

$$
d X(t)=(\beta X(t)+\delta(t)) d t+\sigma X(t) d W(t)+\int_{U} g(X(t-), u) \tilde{N}(d t, d u)
$$

where $X(t-):=\lim _{s \rightarrow t} X(s)$. The integral $\int_{U} g(X(t-), u) \tilde{N}(d t, d u)$ depends on the Poisson measure and is regarded as a jump. Precise assumptions on the data of Equation (1) are given in Section 2.

The remainder of the paper is organized as follows. In the next section, we give the limit theorem and its proof for the stochastic metal price model with jumps. The limit theorem of a two-factor affine model is given in the last section. 


\section{The long-term behavior of stochastic metal price models with jumps}

Let $\left(\Omega, \mathcal{F}_{t}, P\right)$ be a complete probability space in which two mutually independent processes are defined: $\left(W_{t}\right)_{t \geq 0}$ a standard $d$-dimensional Brownian motion and $N$ a Poisson random measure on $(0,+\infty) \times(Z \backslash\{0\})$, where $Z \subset R^{d}$ is equipped with its Borel field $\mathcal{B}_{Z}$, with the Levy compensator $\tilde{N}(d t, d z)=d t v(d z)$, i.e., $\{\tilde{N}((0, t] \times A)=(N-\tilde{N})((0, t] \times A)\}_{t>0}$ is an $\mathcal{F}_{t}$ martingale for each $A \in \mathcal{B}_{Z}$. Hence $v(d z)$ is a Poisson $\sigma$-finite measure satisfying $\int_{Z} v(d z)<\infty$. We assume that there exist a sufficiently large constant $\gamma>0$ and a function $\rho: R^{k} \rightarrow R^{+}$with $\int_{Z} \rho^{2}(z) v(d z)<\infty$ such that

$$
\begin{aligned}
& \left\langle b(t, x)-b\left(t, x^{\prime}\right), x-x^{\prime}\right\rangle \leq \gamma\left|x-x^{\prime}\right|^{2}, \\
& \left|\sigma(t, x)-\sigma\left(t \cdot x^{\prime}\right)\right| \leq \gamma\left|x-x^{\prime}\right|, \\
& |b(t, x)|+|\sigma(t, x)| \leq \gamma(1+|x|), \\
& \left|g(x, z)-g\left(x^{\prime}, z\right)\right| \leq K\left|x-x^{\prime}\right|, \\
& |g(x, z)| \leq \rho(z)(1+|x|),
\end{aligned}
$$

where $\langle\cdot, \cdot \cdot\rangle$ and $|\cdot|$ denote the Euclidean scalar product and the norm, respectively. Obviously, under the above assumptions, there exists a unique strong solution to (1) (see, e.g., [14]).

Lemma 1 If $X(t)$ satisfies (1) and $P(X(0) \geq 0)=1$, then $P(X(t) \geq 0$ for all $t \geq 0)=1$.

Proof Let $a_{0}=1$ and $a_{k}=\exp (-k(k+1) / 2)$ for $k \geq 1$, so that $\int_{a_{k}}^{a_{k-1}} \frac{d u}{u}=k$. For each $k \geq 1$, there clearly exists a continuous function $\psi_{k}(u)$ with support in $\left(a_{k}, a_{k-1}\right)$ such that

$$
0 \leq \psi_{k}(u) \leq \frac{2}{k u} \quad \text { for } a_{k}<u<a_{k-1}
$$

and $\int_{a_{k}}^{a_{k-1}} \psi_{k}(u) d u=1$. Define $\varphi_{k}(x)=0$ for $x \geq 0$ and

$$
\varphi_{k}(x)=\int_{0}^{-x} d y \int_{0}^{y} \psi_{k}(u) d u \quad \text { for } x<0 .
$$

It is easy to observe that $\varphi \in C^{2}(R, R)$ and $-1 \leq \varphi_{k}(x) \leq 0$ if $x<-a_{k}$ or otherwise $\varphi_{k}^{\prime}(x)=0$; $\varphi^{\prime \prime}(x) \leq \frac{2}{k x^{2}}$ if $-a_{k-1}<x<-a_{k}$ or otherwise $\varphi^{\prime \prime}(x)=0$.

Moreover,

$$
x^{-}-a_{k-1} \leq \varphi_{k}(x) \leq x^{-} \text {for all } x \in R,
$$

where $x^{-}=-x$ if $x<0$ or otherwise $x^{-}=0$.

For any $t \geq 0$, by Ito's formula, we can derive

$$
\begin{aligned}
E \varphi_{k}(X(t))= & E \varphi_{k}\left(X_{0}\right)+E \int_{0}^{t} \varphi_{k}^{\prime}(X(s))\{\beta X(s)+\delta(s)\} d s \\
& +\frac{\sigma^{2}}{2} E \int_{0}^{t} \varphi^{\prime \prime}(X(s)) X^{2}(s) d s
\end{aligned}
$$




$$
\begin{aligned}
& +E \int_{0}^{t} \int_{Z}\left\{\varphi_{k}(X(s)+g(X(s), u))-\varphi_{k}(X(s))\right. \\
& \left.-\varphi_{k}^{\prime}(X(s)) g(X(s), u)\right\} n(d u) d s \\
\leq & \frac{C_{1}}{k}+E \int_{0}^{t} \int_{Z} \int_{0}^{1}\left(\varphi_{k}^{\prime}\left(\theta g\left(\left(X_{s}\right), u\right)+X(s)\right)-\varphi_{k}^{\prime}(X(s))\right) \\
& \times g(X(s), u) d \theta n(d u) d s \\
\leq & \frac{C_{1}}{k}+C_{2} n^{\frac{1}{2}}(Z) E \int_{0}^{t} X^{-}(s) I_{X(s) \leq 0} d s \\
\leq & \frac{C_{1}}{k} C_{2} n^{\frac{1}{2}}(Z) a_{k-1} t+C_{2} n^{\frac{1}{2}}(Z) \int_{0}^{t} E \varphi_{k}(X(s)) d s .
\end{aligned}
$$

Combining with the Gronwall inequality, we have

$$
E X^{-}(t)-a_{k-1} \leq E \varphi_{k}(X(t)) \leq C\left(\frac{1}{K}+a_{k-1}\right) .
$$

Hence $E X^{-}(t)=0$ as $k \rightarrow \infty$ and the nonnegative property of the solution $\{X(t)\}_{t \geq 0}$ follows.

Lemma 2 Let $\beta<0$ and assume that $2 \beta+K^{2}<0$. Then there exist $k>0$ and $C>0$ such that

$$
E\left(e^{-k \beta \tau} X^{2}(\tau)\right) \leq C_{1}+C_{2} E \int_{0}^{\tau} e^{-k \beta s}\left(\delta^{2}(s)+1\right) d s,
$$

where $\tau>0$ is a bounded stopping time.

Proof By Ito's formula, we have

$$
\begin{aligned}
d\left(e^{-k \beta t} X^{2}(t)\right)= & e^{-k \beta t}\left\{(2-k) \beta X^{2}(t)+\sigma^{2} X^{2}(t)+2 \delta(t) X(t)\right. \\
& \left.+\int_{U} g^{2}(X(t), u) n(d u)\right\} d t+2 \sigma e^{-k \beta t} X^{2}(t) d W(t) \\
& +e^{-k \beta t} \int_{U}\left(g^{2}(X(t), u)+2 X(t) g(X(t), u)\right) \tilde{N}(d t, d u) .
\end{aligned}
$$

Integrating from 0 to $\tau$ and taking expectations on both sides, we have

$$
\begin{aligned}
E\left(e^{-k \beta \tau} X^{2}(\tau)\right) \leq & C_{1}+\left((2-k) \beta+2 \epsilon+K^{2}\right) E \int_{0}^{\tau} e^{-k \beta s} X^{2}(s) d s \\
& +C_{2} E \int_{0}^{\tau}\left(\delta^{2}(s)+1\right) d s,
\end{aligned}
$$

where $\epsilon>0$ is arbitrary. Due to $2 \beta+K^{2}<0$, we choose $K>0$ and $\epsilon$ such that $(2-k) \beta+$ $2 \epsilon+K^{2}=0$.

Now we turn to the proof of the convergence theorem. 
Theorem 1 Let $X(t)$ be a solution to (1) and assume that there is $\mu \geq 1$ and a nonnegative random variable $\bar{\delta}$ such that

$$
\lim _{t \rightarrow \infty} \frac{1}{t^{\mu}} \int_{0}^{t} \delta(s) d s \rightarrow \bar{\delta} \quad \text { a.s. }
$$

Then the following convergence holds:

$$
\frac{1}{t^{\mu}} \int_{0}^{t} X(s) d s \rightarrow-\frac{\bar{\delta}}{\beta} \quad \text { a.s. as } t \rightarrow \infty \text {. }
$$

Proof We use Kronecker's lemma in [12].

Dividing equation (1) by $\beta(1+t)^{\mu}$ gives the equality

$$
\begin{aligned}
& \frac{1}{(1+t)^{\mu}} \int_{0}^{t}\left(X(s)+\frac{\delta(s)}{\beta}\right) d s \\
& \quad=\frac{X(t)-X(0)}{\beta(1+t)^{\mu}}-\int_{0}^{t} \frac{X(s)}{\beta(1+t)^{\mu}} d B(s)-\int_{0}^{t} \int_{U} \frac{g(X(s-), u)}{\beta(1+t)^{\mu}} \tilde{N}(d s, d u) .
\end{aligned}
$$

Let us introduce the sequence $\left(T_{n}\right)_{n \geq 1}$ of stopping times

$$
T_{n}=\inf \left\{t \geq 0 \mid \int_{0}^{t} \frac{\delta^{2}(u)}{(u+1)^{2}} d u \geq n\right\}
$$

Since by hypothesis $\frac{1}{s+1} \int_{0}^{s} \delta_{u} d u \rightarrow \bar{\delta}$ a.s., we obtain that $\int_{0}^{u} \delta_{s} d s \leq K(u+1)$ a.e. for some constant $K$ depending on $\omega$. A straightforward calculation shows that

$$
\int_{0}^{\infty} \frac{\delta^{2}(u)}{u+1} d u<\infty \quad \text { a.s. }
$$

Hence $\left\{T_{n}=\infty\right\} \uparrow \Omega$, and consequently, we only need to prove the existence a.e. of $\int_{0}^{\infty} \frac{g X_{u}^{T_{n}}}{u+1} d B_{u}$ on $\{T=\infty\}$, where $g(x)=x$.

Moreover, since $\int_{0}^{\infty} \frac{X_{u}^{T_{n}}}{u+1} d B_{u}$ is a local martingale, it suffices to remark that $\int_{0}^{t} \frac{X_{u}^{T_{u}}}{u+1} d B_{u}$ is an $L^{2}$ bounded martingale,

$$
\left\|\int_{0}^{t} \frac{X_{u}^{T_{n}}}{u+1} d B_{u}\right\|_{2}^{2}=\int_{0}^{t} E\left(X_{u}^{T_{n}}\right)^{2} \frac{1}{(u+1)^{2}} d u .
$$

In order to evaluate the integral, we remark that

$$
\begin{aligned}
E\left(X_{u}^{T_{n}}\right)^{2} & \leq e^{2 \beta u} E\left[e^{-2 \beta u} X_{u}^{2} I_{u \leq T_{n}}\right] \\
& \leq e^{2 \beta u} E\left[e^{-2 \beta\left(u \wedge T_{n}\right)} X_{u \wedge T_{n}}^{2}\right] .
\end{aligned}
$$

In Lemma 2, we have obtained the inequality

$$
E\left(e^{-k \beta \tau} X^{2}(\tau)\right) \leq C_{1}+C_{2} E \int_{0}^{\tau} e^{-k \beta s}\left(\delta^{2}(s)+1\right) d s .
$$


Consequently,

$$
\begin{aligned}
E\left(X_{u}^{T_{n}}\right)^{2} & \leq e^{2 \beta u}\left(X_{0}+E \int_{0}^{u \wedge T_{n}} e^{-2 \beta s}(\delta(s)+1) d s\right) \\
& \leq X_{0} e^{2 \beta u}+e^{2 \beta u} \int_{0}^{u} e^{-2 \beta s} E\left[\delta^{2}(s) I_{\left(s \leq T_{n}\right)}\right] d s .
\end{aligned}
$$

Using this result, we obtain

$$
\begin{aligned}
& \int_{0}^{t} E\left(X_{u}^{T_{n}}\right)^{2} \frac{1}{(u+1)^{2}} d u \\
& \quad \leq \int_{0}^{t} X_{0} \frac{e^{2 \beta u}}{(u+1)^{2}} d u+\int_{0}^{t} X_{0} \frac{e^{2 \beta u}}{(u+1)^{2}} d u \int_{0}^{u} e^{-2 \beta s} E\left[\delta_{s}^{2} I_{\left(s \leq T_{n}\right)}\right] d s .
\end{aligned}
$$

Obviously, the first term is uniformly bounded in $t$. For the second term, we apply Fubini's theorem to find a bound which does not depend on $t$,

$$
\begin{aligned}
& \int_{0}^{t} X_{0} \frac{e^{2 \beta u}}{(u+1)^{2}} d u \int_{0}^{u} e^{-2 \beta s} E\left[\delta_{s}^{2} I_{\left(s \leq T_{n}\right)}\right] d s \\
& \quad=\int_{0}^{t} e^{-2 \beta s} E\left[\delta_{s}^{2} I_{\left(s \leq T_{n}\right)}\right] d s \int_{s}^{t} \frac{e^{2 \beta u}}{(u+1)^{2}} d u \\
& \quad \leq \int_{0}^{t} E\left[\delta_{s}^{2} I_{\left(s \leq T_{n}\right)}\right] \frac{1}{(s+1)^{2}} \frac{-1}{2 \beta} d s \\
& \quad \leq \frac{-1}{2 \beta} E\left[\int_{0}^{T_{n}} \frac{\delta_{s}^{2}}{(s+1)^{2}} d s\right] \\
& \quad \leq-\frac{n}{2 \beta} .
\end{aligned}
$$

The third term converges to 0 by observing that

$$
\begin{aligned}
& E\left(\int_{0}^{t} \int_{U} \frac{g(X(s), u)}{(1+s)^{\mu}} I_{\left(s \leq \tau_{n}\right)} \tilde{N}(d s, d u)\right)^{2} \\
& =E \int_{0}^{t} \int_{U} \frac{g^{2}(X(s), u)}{(1+s)^{2 \mu}} I_{\left(s \leq \tau_{n}\right)} v(d u) d s .
\end{aligned}
$$

\section{The long-time behavior of affine models}

As an application of Theorem 1, we consider the long-time behavior of an affine model in a two-dimensional case,

$$
\begin{aligned}
& d X(t)=\left(\beta_{1} X(t)+\delta(t)\right) d t+\sigma_{1} X(t) d W_{1}(t)+X(t) \int_{U} u \tilde{N}_{1}(d t, d u), \\
& d Y(t)=\left(\beta_{2} Y(t)+X(t)\right) d t+\sigma_{2} Y(t) d W_{2}(t)+Y(t) \int_{U} u \tilde{N}_{2}(d t, d u),
\end{aligned}
$$

where $\beta_{1}<0$ and $\beta_{2}<0$. Let $\left(\Omega,\left(\mathcal{F}_{t}\right)_{t \geq 0}, P\right)$ be a filtered probability space satisfying the usual hypothesis. Suppose that on this probability space the following objects are defined:

(i) a two-dimensional Brownian motion $W(\cdot)=\left(W_{1}(\cdot), W_{2}(\cdot)\right)$; 
(ii) $N_{1}(d t, d u), N_{2}(d t, d u)$ represent Poisson counting measures with characteristic measures $v_{1}(\cdot)$ and $v_{2}(\cdot)$, respectively.

For model (2), we are interested in the almost sure convergence of the long-term behavior $t^{-\mu} \int_{0}^{t} Y(s) d s$ for some $\mu \geq 1$.

The process $Y(t)$ has a reversion level $X(t)$ which is a stochastic process itself. From Dawson and $\mathrm{Li}[15]$, the equation system has a unique strong solution $(X(\cdot), Y(\cdot))$. Moreover, $(X(\cdot), Y(\cdot))$ is an affine Markov process. Now, we give the main theorem of this section.

Theorem 2 Assume that $(X(\cdot), Y(\cdot))$ is a solution to the equation system (2). Then we have

$$
\frac{1}{t^{\mu}} \int_{0}^{t} Y(s) d s \rightarrow-\frac{\bar{\delta}}{\beta_{1} \beta_{2}} \quad \text { a.s. as } t \rightarrow \infty \text {. }
$$

Proof It can be obtained by similar arguments as Theorem 1.

Another application of Theorem 1 is that if the average of the drift converges almost surely to a constant, then the long-term trend of the model will revert to a line almost surely.

Corollary 3 Let $\delta: \omega \times R_{+} \rightarrow R_{+}$and there exist constants $\mu \geq 1$ and $\bar{\delta} \geq 0$ such that

$$
\lim _{t \rightarrow \infty} \frac{1}{t^{\mu}} \int_{0}^{t} \delta(s) d s \rightarrow \bar{\delta} \quad \text { a.s. }
$$

Then the following convergence holds for equation (1):

$$
\frac{1}{t^{\mu}} \int_{0}^{t} X(s) d s \rightarrow-\frac{\bar{\delta}}{\beta} \quad \text { a.s. as } t \rightarrow \infty .
$$

In Figure 2, the long-term behavior of the model is plotted with $\mu=\beta=\sigma=1$ and $\bar{\delta}=0$.

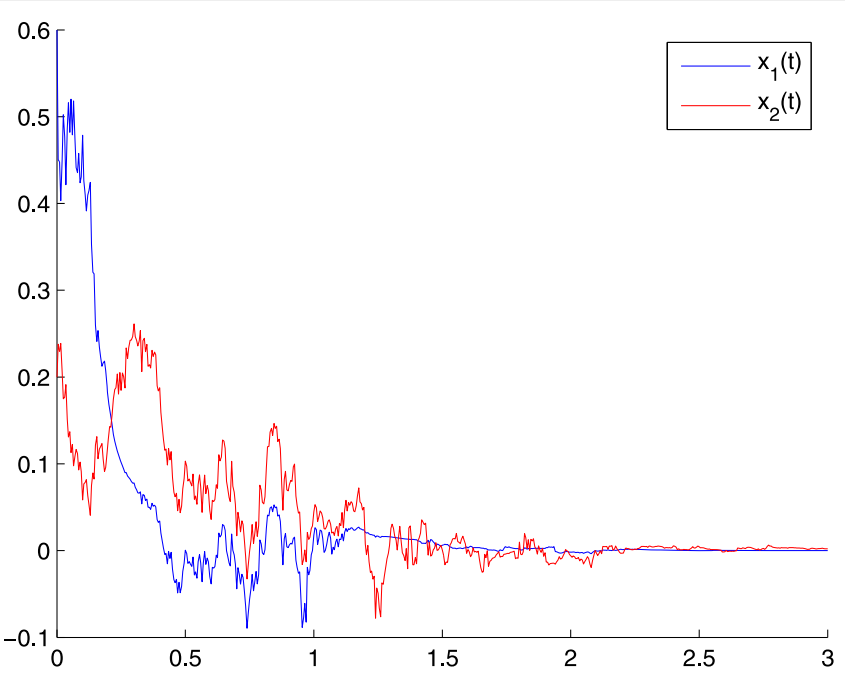

Figure 2 Numerical result for Corollary 3 with $\bar{\delta}=0$. 


\section{Competing interests}

The authors declare that they have no competing interests.

\section{Authors' contributions}

All authors completed the paper together. All authors read and approved the final manuscript.

\section{Author details}

${ }^{1}$ Business School, Central South University, Changsha, Hunan 410083, China. ${ }^{2}$ School of Mathematics and Statistics, Central South University, Changsha, Hunan 410083, China. ${ }^{3}$ Institute of Metal Resources Strategy, Central South University, Changsha, Hunan 410083, China.

\section{Acknowledgements}

This work was supported by the Postdoctoral Foundation of Central South University, Major Program of the National Social Science Foundation of China (13\&ZD024), the Hunan Provincial Natural Science Foundation of China (14JJ3019) and the National Natural Science Foundation of China (Grant No. 11101433).

Received: 20 January 2014 Accepted: 17 July 2014 Published: 4 August 2014

\section{References}

1. Ahrens, WA, Sharma, VR: Trends in natural resource commodity prices: deterministic or stochastic? J. Environ. Econ. Manag. 33, 59-74 (1997)

2. Arellano, C, Pantula, SG: Testing for trend stationarity versus difference stationarity. J. Time Ser. Anal. 16, 147-164 (1995)

3. Lee, J, List, JA, Strazicich, MC: Non-renewable resource prices: deterministic or stochastic trends? J. Environ. Econ. Manag. 51, 354-357 (2006)

4. Pindyck, RS: The long-run evolution of energy prices. Energy J. 20, 1-27 (1999)

5. Shahriar, S, Erkan, T: A long-term view of worldwide fossil fuel prices. Appl. Energy 87, 988-1000 (2010)

6. Brennan, MJ, Schwartz, ES: Evaluating natural resource investment. J. Bus. 58, 135-157 (1985)

7. Choi, K, Hammoudeh, S: Volatility behavior of oil, industrial commodity and stock markets in a regime-switching environment. Energy Policy 38, 4388-4399 (2010)

8. Roberts, M: Duration and characteristics of metal price cycles. Resour. Policy 34, 87-102 (2009)

9. Hamilton, J: Time Series Analysis. Princeton University Press, Princeton (1994)

10. Harding, D, Pagan, A: A comparison of two business cycle dating methods. J. Econ. Dyn. Control 27, $1681-1690$ (2002)

11. Bao, J, Yuan, C: Long-term behavior of stochastic interest rate with jumps. Insur. Math. Econ. 53, 266-272 (2013)

12. Deelstra, G, Delbaen, F: Long-term returns in stochastic interest rate models. Insur. Math. Econ. 17, 163-169 (1995)

13. Zhao, J: Long time behavior of stochastic interest rate models. Insur. Math. Econ. 44, 459-463 (2009)

14. Applebaum, D: Lévy Processes and Stochastic Calculus. Cambridge University Press, Cambridge (2004)

15. Dawson, DA, Li, ZH: Skew convolution semigroup and affine Markov processes. Ann. Probab. 34, 1103-1142 (2006)

\section{Submit your manuscript to a SpringerOpen ${ }^{\ominus}$ journal and benefit from:}

- Convenient online submission

- Rigorous peer review

- Immediate publication on acceptance

- Open access: articles freely available online

- High visibility within the field

- Retaining the copyright to your article 